\title{
Alliances to Disseminate Addiction Prevention and Treatment (ADAPT): Assessing the Exchange of Ideas in Alliances Between Juvenile Justice and Community Mental Health Centers
}

\author{
Elise Patrick ${ }^{1}$, Katherine Schwartz ${ }^{2}$, Fangquian Ouyang ${ }^{3}$, Stanley Taylor ${ }^{3}$, Matthew Aalsma ${ }^{2}$ \\ ${ }^{1}$ Indiana University School of Medicine; ${ }^{2}$ Indiana University School of Medicine, Department of \\ Pediatrics; ${ }^{3}$ Indiana University School of Medicine, Department of Biostatistics and Health Data \\ Science
}

\section{Background/Hypothesis:}

In the US, over half of youth involved in the juvenile justice system meet criteria for substance use disorder (SUD). Further, SUD is a consistent predictor of recidivism. Thus, significant improvements are needed to assure that justice-involved youth who meet criteria for SUD are screened and referred to care, especially in rural settings. ADAPT, an ongoing, statewide project, employs a learning health system (LHS) model to implement evidence-based practices (EBPs) to develop and improve alliances between juvenile justice $(\mathrm{JJ})$ and community mental health centers (CMHCs). We assessed collaboration, "alliance," between these systems.

\section{Methods:}

The LHS alliance was assessed with self-report surveys distributed to personnel at 8 county sites. These included the cultural exchange inventory (CEI) measure to assess the process of exchanges in knowledge (beliefs about the process of implementing EBPs), and outcomes of those exchanges (beliefs about the outcome of interagency collaboration), to assess the alliance between $\mathrm{JJ}$ and $\mathrm{CMHCs}$.

\section{Results:}

Pre-implementation surveys indicated a significant difference between $\mathrm{CMHC}$ respondents (CEI Output $\mathrm{M}=3.55, \mathrm{SD}=1.53)$ compared to $\mathrm{JJ}$ respondents $(\mathrm{M}=2.89, \mathrm{SD}=1.57)$ about the outcomes of the exchange of ideas [t(190) $=2.13, p=0.03]$. Both CMHC (CEI Process $M=3.93, S D=1.45$ ) and JJ participants (CEI Process $\mathrm{M}=3.84, \mathrm{SD}=1.96$ ) reported similar beliefs about the process of idea exchange $\mathrm{t}(119)=0.27, p=0.78$ ). Further, participants from low rurality counties (i.e., more urban) reported less favorably to beliefs about the outcomes of collaboration (CEI Output $M=3.16, S D=1.62$ ) compared to medium rurality county participants $[M=3.76, S D=1.39$; $t(120)=2.03, p=0.04]$. Thus, we find a difference in perception of collaboration both between systems and between counties of varying rurality.

\section{Conclusion/Potential Impact:}

These findings help capture the current barriers to collaboration that exist between $\mathrm{JJ}$ and CMHCs before implementation of the LHS framework. Understanding these barriers between systems is essential to better cultivate interagency alliances to improve care for justice-involved youth with SUDs. 